\title{
Application and Implementation of Image Point Operation in Gray Scale Transformation
}

\author{
Zhang Hongmei \\ College of Computer Science and Technology \\ Inner Mongolia University For Nationalities \\ TongLiao, China \\ e-mail:Tongyu_007@163.com
}

\author{
Zhang Zhigao \\ College of Math \\ Inner Mongolia University For Nationalities \\ TongLiao, China \\ e-mail: zzgtongxin@163.com
}

\author{
Pei Zhili* \\ College of Computer Science and Technology \\ Inner Mongolia University For Nationalities \\ TongLiao, China \\ e-mail:zhilipei@sina.com \\ *corresponding author
}

\begin{abstract}
Gray scale transformation is a common image enhancement technology in digital image processing. It can facilitate operations such as strengthening and stretching image contrast, layering gray levels, and inversing pictures so as to highlight image characters and image contrast. Image point operation is a preferable method to transform grey level of images and with various transformation functions, image point operation can be categorized linear functions and non-linear ones. The article sums up circumstances where common point operations are applied and conducts simulation experiment by using MATLAB software before it provides the experiment results.
\end{abstract}

Keywords-gray scale transformation of images; image point operation; matlab

\section{INTRODUCTION}

The process used to be called the spatial transformation of the image, is to realize the image processing by the spatial function transformation. It is the most common technology of image enhancement in digital image processing. The pixels are operated by various functions by using the space of the image pixels [1]. There are two ways to realize the spatial transformation: The first one is based on pixels. What we call Image point operation is that each of the image processing is performed on each pixel, and has nothing to do with other pixels. The second one is based on template. What we call template operation or domain operation is that the process of image processing is to deal with each sub graph of the image. Each sub graph is a geometric neighborhood of the center of a certain pixel. This paper focuses on the analysis and introduction of image point operation on the image gray transformation, and the role of the MATLAB programming to achieve the image point operation, the experimental results are given by the experimental effects ${ }^{[2]}$.

\section{IMAGE POINT OPERATION}

The image point operations refers to every pixel in the image is calculated, so that the output of each pixel value are only decided by the corresponding point values, which can be understood as a mapping between point-to-point ${ }^{[3]}$. The function form is $\mathrm{B}(\mathrm{x}, \mathrm{y})=\mathrm{f}[\mathrm{A}(\mathrm{x}, \mathrm{y})]$, where $\mathrm{A}(\mathrm{x}, \mathrm{y})$ is the input images and $\mathrm{B}(\mathrm{x}, \mathrm{y})$ is the output images and $\mathrm{f}$ is map function ${ }^{[4]}$. Point operation, commonly referred to as gray scale transformation, is one of the common methods of image enhancement processing. It is often used to change the gray scale and distribution of the image ${ }^{[5]}$. By this method, the dynamic range of the image can be increased, the contrast of the image can be enhanced and the image becomes more distinct.

The point operation is determined by the gray level mapping function $\mathrm{f}$. According to the difference of $\mathrm{F}$, the operation can be divided into two categories, linear point operation and nonlinear point operation ${ }^{[6]}$.

The function form of linear point operation can be described by linear equation: $F(D A)=a * D A+b=D B$, where DA is the gray value of input points and DB is used to indicate the gray value of the corresponding output points.

The so-called nonlinear point operation is that the mapping function $\mathrm{f}$ is a nonlinear relationship. The most common of nonlinear relationship are the logarithm and power index, etc ${ }^{[7]}$. The general form of the logarithmic transformation is: $\mathrm{D}^{\prime}=\mathrm{c}^{*} \log (1+|\mathrm{D}|)$, where $\mathrm{c}$ is a constant.

Using a logarithmic function to complete the nonlinear transform of the image, so as to realize gray level of image expansion and compression, it can map the low gray values in a narrower range of the image to a wider range of gray values ${ }^{[8]}$. Similarly, the same reason for the high gray values. And the general form of the power exponent is $\mathrm{s}=\mathrm{c} \mathrm{r}{ }^{\gamma}$, where $\mathrm{C}$ and $\gamma$ are 
nonnegative constants ${ }^{[9]}$. The function diagram is shown in Fig.1.

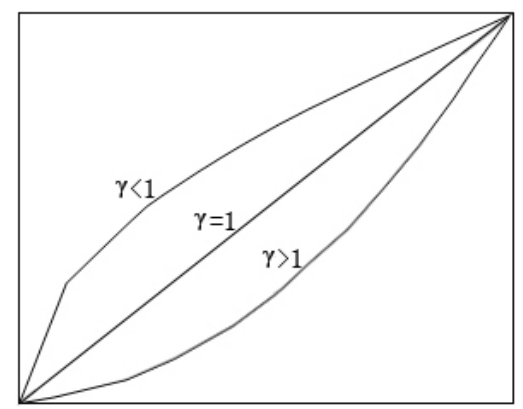

Fig.1. Sketch for $\mathrm{s}=\mathrm{cr}^{\gamma}$

It can be known by analyzing figure 1 that the power exponent transformation is similar to the logarithmic transformation when $\gamma<1$; And for $\gamma>1$ the situation is just the reverse. But when $\gamma=1$ and $\mathrm{c}=1$, the situation is identity transformation. By comparison, we can know that the power exponent is used wider than the logarithmic transformation, and the image contrast enhancement of the power exponent transform is conventionally called gamma correction.

\section{REALIZATION OF IMAGE POINT OPERATION BASED ON MATLAB}

Due to the limitation of space, this paper will focus on two examples, which are respectively selected from the linear point operation and the nonlinear point operation.

Basic ideas: First, construct function of contrast enhancement. Then, every pixel in the image is transformed using double loop control by a linear transformation or nonlinear transformation respectively [10]. Finally, the reconstructed image and the original image are compared and analyzed, and the experimental results are given.

(1) Code for linear point operations is as follows:

Linear point operation of form $\mathrm{DB}=\mathrm{a} * \mathrm{DA}+\mathrm{b}$ :

x1=imread('pout.tif') ;

subplot $(2,3,1)$;

imshow(x1),title('Original image') ;

axis square

$\mathrm{f} 0=0 ; \mathrm{g} 0=30$;

$\mathrm{fl}=255 ; \mathrm{g} 1=255$;

subplot $(2,3,2)$;

$\operatorname{plot}([\mathrm{f} 0, \mathrm{fl}],[\mathrm{g} 0, \mathrm{~g} 1])$;

title(' Transformation curves for enhanced contrast');

axis square ;

$\operatorname{axis}([0,256,0,256])$

grid on

$\mathrm{a}=(\mathrm{g} 1-\mathrm{g} 0) /(\mathrm{f} 1-\mathrm{f0})$; $[\mathrm{m}, \mathrm{n}]=\operatorname{size}(\mathrm{x} 1)$;

$\mathrm{x} 2=\operatorname{double}(\mathrm{x} 1)$;

for $\mathrm{i}=1: \mathrm{m}$

$$
\begin{aligned}
& \text { for } \mathrm{j}=1: \mathrm{n} \\
& \qquad \begin{array}{l}
\mathrm{f}=\mathrm{x} 2(\mathrm{i}, \mathrm{j}) ; \\
\mathrm{g}(\mathrm{i}, \mathrm{j})=0 ; \\
\mathrm{if}(\mathrm{f}>=0) \&(\mathrm{f}<=255) \\
\quad \mathrm{g}(\mathrm{i}, \mathrm{j})=\mathrm{a} * \mathrm{f}+\mathrm{g} 0 ;
\end{array}
\end{aligned}
$$

end

end

end

subplot(2,3,3), imshow(mat2gray(g));

title(' Transformed image ') ;

axis square

In order to verify the effect of the treatment, the A and B were taken to different values, and the following results were obtained:

1) When $a=1.5 b=20$, the processing effect is shown in Fig. 2.

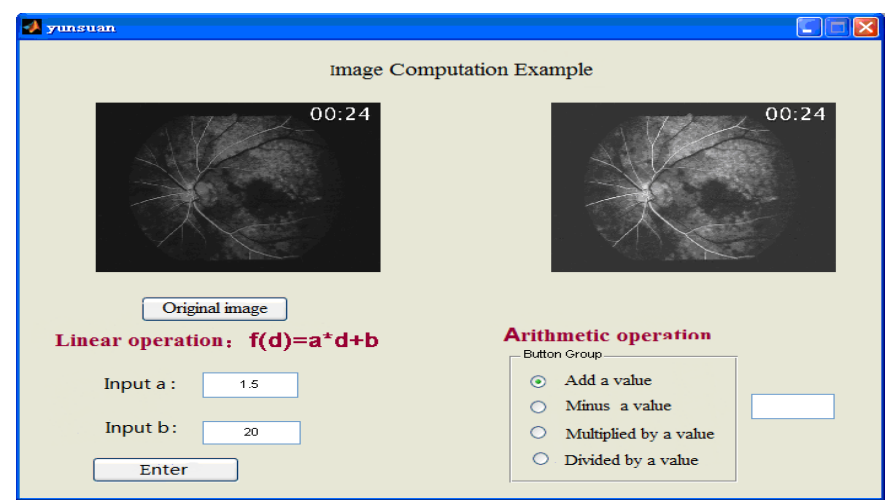

Fig. 2 Comparison chart of linear point operation after $\mathrm{a}=1.5$

2) When $a=0.5 b=20$, the processing effect is shown in Fig. 3.

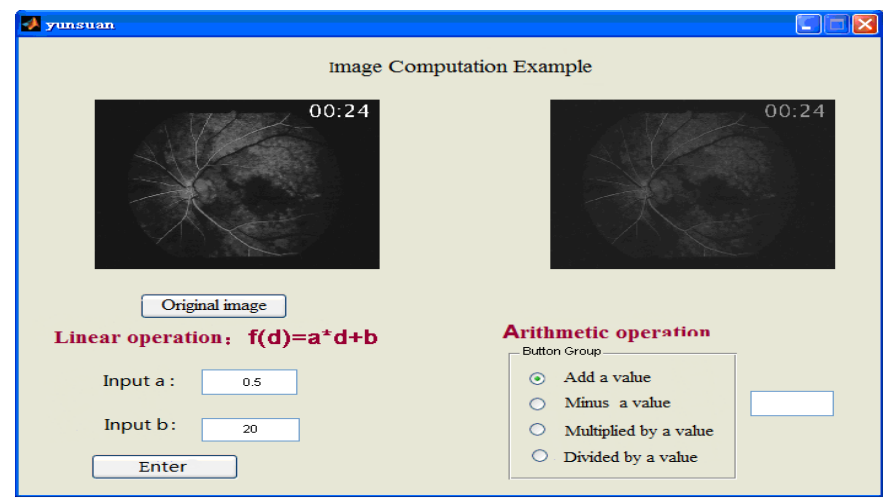

Fig. 3 Comparison chart of linear point operation after $\mathrm{a}=0.5$ 
3) When $a=1 \quad b=0$, the processing effect is shown in Fig.4.

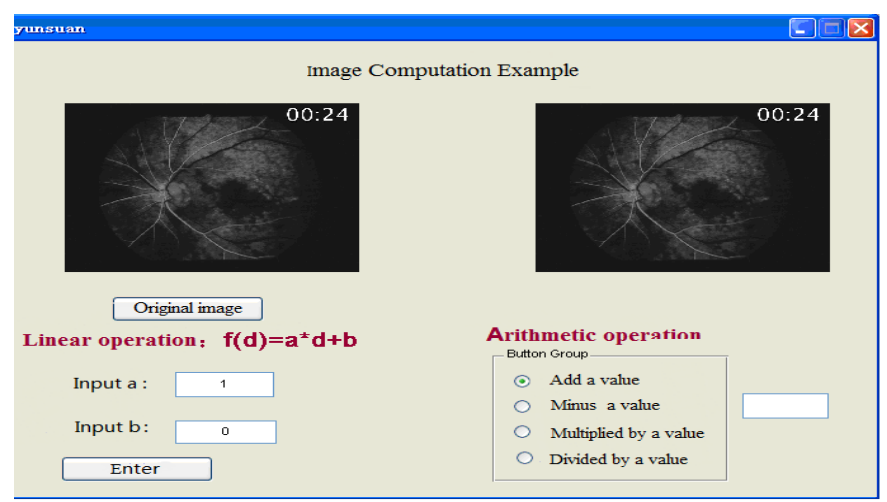

Fig. 4 Comparison chart of linear point operation after $\mathrm{a}=1$

4) When $a=-1 \quad b=100$, the processing effect is shown in Fig.5.

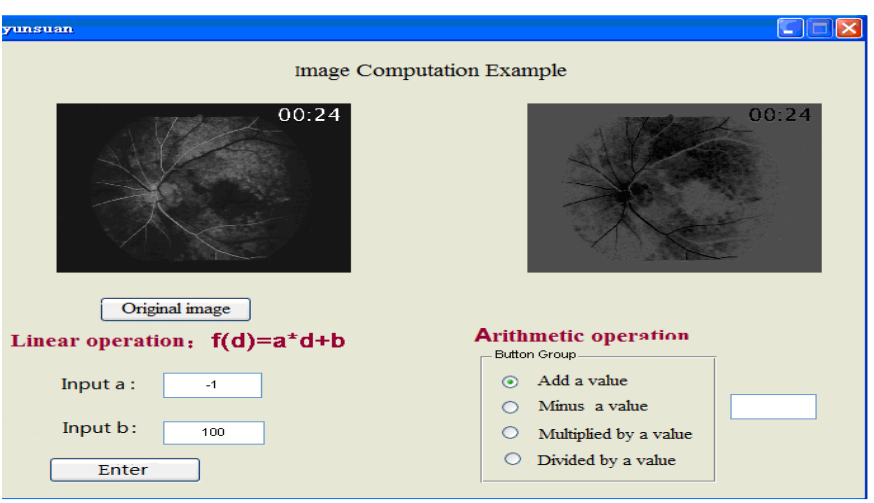

Fig. 5 Comparison chart of linear point operation after $\mathrm{a}=-1$

(2) Code for nonlinear point operations is as follows:

Linear point operation of form $\mathrm{D}^{\prime}=\mathrm{c}^{*} \log (1+|\mathrm{D}|)$

$\mathrm{x} 1=$ imread('circuit.tif');

subplot $(2,3,1)$;

imshow(x1),title('Original image');

axis square

$\mathrm{c}=255 / \log (256)$;

$\mathrm{x}=0: 1: 255$;

$\mathrm{y}=\mathrm{c} * \log (1+\mathrm{x})$;

subplot $(2,3,2)$;

$\operatorname{plot}(\mathrm{x}, \mathrm{y})$

title(' Transformation curves for enhanced contrast ');

axis square;

$\operatorname{axis}([0,256,0,256])$

grid on

$\mathrm{r}=(\mathrm{g} 1-\mathrm{g} 0) /(\mathrm{fl}-\mathrm{f} 0)$;

$[\mathrm{m}, \mathrm{n}]=\operatorname{size}(\mathrm{x} 1)$; $\mathrm{x} 2=\operatorname{double}(\mathrm{x} 1)$

for $\mathrm{i}=1: \mathrm{m}$

$$
\begin{aligned}
& \text { for } \mathrm{j}=1: \mathrm{n} \\
& \qquad \mathrm{g}(\mathrm{i}, \mathrm{j})=\mathrm{c}^{*} \log (\mathrm{x} 2(\mathrm{i}, \mathrm{j})+1) ;
\end{aligned}
$$

end

end

subplot $(2,3,3)$,imshow(mat2gray $(\mathrm{g}))$;

title(' Transformed image ');

axis square

The processing effect is shown in Fig.6.

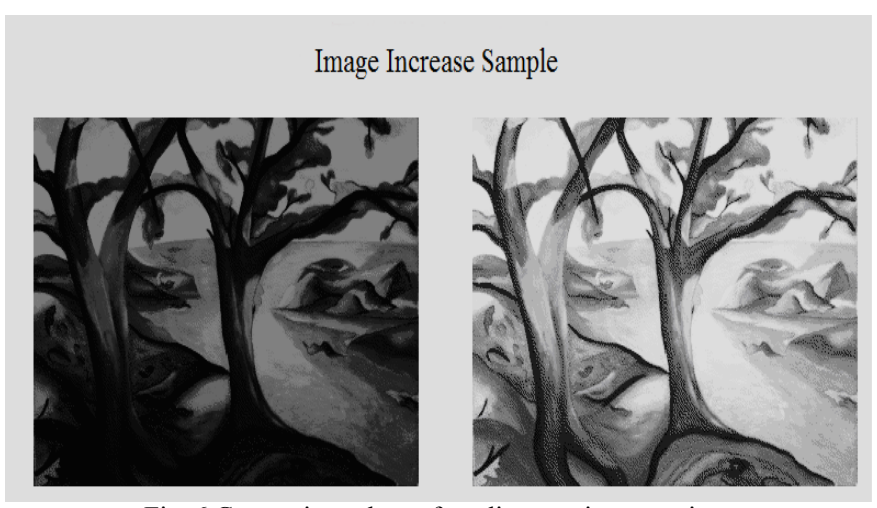

Fig. 6 Comparison chart of nonlinear point operations

\section{CONCLUSIONS}

(1) Through the analysis of the figures, it can be seen that the contrast of the image after the process has been significantly improved. After giving different values of $a$ and $b$, the experimental conclusions can be summarized as follows:

When a $>1$, compared with the original image, the contrast of the processed image has an obvious increase;

When $\mathrm{a}<1$, after the processing, the contrast of the image is significantly decreased;

When $a=1$ and $b=0$, there is no difference between the processed image and the original image, and only simple copy is realized;

When $\mathrm{a}=1$ and $\mathrm{b}>0$, after the processing, the brightness of the image is significantly enhancement;

When $\mathrm{a}=1$ and $\mathrm{b}<0$, after the processing, the brightness of the image is reduced;

When $\mathrm{a}=-1$ and $\mathrm{b}=0$, produces a negative image;

When $b>0$, the brightness of the image will be increased;

When $b<0$, the brightness of the image will be reduced.

(2) By the analysis in Figure 6 can be seen that, Pixels in the original image of low gray-scale range are transformed into the high gray level, so that the whole image gray level has been significantly improved. Therefore, the image gray level of the image can be expanded and compressed by using the nonlinear transformation, such as logarithmic or exponential. 


\section{ACKNOWLEDGMENT}

This work was financially supported by the National Natural Science Foundation of China (61163034, 61373067), the Grassland Excellent Talents Project of Inner Mongolia Autonomous Region (2013), the supported By Program for Young Talents of Science and Technology in Universities of Inner Mongolia Autonomous Region(NJYT-14-A09), the Inner Mongolia Natural Science Foundation(2013MS0911), the 321 Talents Project the two level of Inner Mongolia Autonomous Region(2010), the Inner Mongolia talent development fund(2011), and the Scientific Research Foundation of Inner Mongolia University For Nationalities(NMDYB15011)

\section{REFERENCES}

[1] HU Qiong,WANG Ronggui,HU Weiwei,YANG Wanting. Color Image Enhancement Based on Histogram Segmentation[J]. Journal of Image and Graphics,2009,14(9):1776-1781.

[2] LI Guanzhang,LUO Wusheng,LI Pei. Color Image Enhancement Based on Visual Characteristics of Human Eyes[J]. Opto-Electronic Engineering,2009,36(11):92-95.
[3] Yoon Byoungwoo,Song Woojin.Image Contrast Enhancement Based on the Teneralized Histogram[J].Journal of Electronic Imaging,2007,16(3):033005-033005-8.

[4] GONZALEZ RC,WOODS R E,EDDIN S S L. Digital Image Processing using MATLAB [M].Beijing: Publishing House Of Electronics Industry,2004.

[5] Zhang Yi,Liu Xu,Li Haifeng. Adaptive Image Histogram Equalization Algorithm [J].Journal of ZheJiang University (Engineering Science) .2007,Vol. 41 No.4

[6] Rafael C. Gonzalez, Richard E. Woods. Digital Image Processing(Third Edition)[M].Ruan Qiuqi, translation. Beijing: Publishing House Of Electronics Industry,2011.6:72-77.

[7] Wang Qiwei. Research on image histogram feature and its application[D].University of Science and Technology of China,2014.

[8] Ruan Qiuqi. Digital Image Processing [M]. Beijing: Publishing House Of Electronics Industry,2001.

[9] Castleman Kenneth R. Digital Image Processing[M].Zhu Zhigang, translation.Beijing: Publishing House Of Electronics Industry,1998.

[10] Zheng Yongguo. Research and Implementation on image enhancement methods [D]. Shandong University of Science and Technology,2005. 